\title{
An Active Sampler for Monitoring Polychlorinated Dibenzo-p-dioxins and Furans in Ambient Air
}

\author{
Bing Zhang $\cdot$ Lifei Zhang $\cdot$ Jiajia Wu • \\ Guorui Liu $\cdot$ Minghui Zheng
}

Received: 5 January 2011/Accepted: 15 April 2011/Published online: 24 April 2011

(C) Springer Science+Business Media, LLC 2011

\begin{abstract}
An active sampler for monitoring polychlorinated dibenzo- $p$-dioxins and furans ( $\mathrm{PCDD} / \mathrm{Fs})$ in air was developed using an automobile air filter set. The performance of the mobile air sampler was evaluated. Recoveries of PCDD/F congeners ranged from $62.3 \%$ to $64.5 \%$ with sampling volumes of $500-1,800 \mathrm{~m}^{3}$. Air samples were collected between April and June, 2007 in Beijing. The monitoring results of PCDD/F levels by the mobile sampler were comparable to results obtained by a conventional stationary high-volume active sampler. The results suggest this mobile air sampling technology is a promising new strategy for the assessment of air quality in urban areas.
\end{abstract}

Keywords Active air sampler - Automobile air filter set . PCDD/Fs - Air quality

Polychlorinated dibenzo-p-dioxins (PCDDs) and polychlorinated dibenzofurans (PCDFs) are well-known anthropogenic compounds that are formed during various combustion and industrial processes. Due to their persistence and semi-volatility, PCDD/Fs can be transported over long distances. Therefore, atmospheric transport is a

B. Zhang $\cdot$ L. Zhang $\cdot$ J. Wu $\cdot$ G. Liu $\cdot$ M. Zheng $(\bowtie)$

State Key Laboratory of Environmental Chemistry and Ecotoxicology, Research Center for Eco-Environmental Sciences, Chinese Academy of Sciences, No.18 Shuangqing Road, Haidian District, 100085 Beijing, China

e-mail: zhengmh@rcees.ac.cn

\section{Zhang}

State Environmental Protection Key Laboratory of Dioxin Pollution Control, National Research Center for Environmental Analysis and Measurement, No. 1 Yuhui Nanlu, Chaoyang District, 100029 Beijing, China primary pathway for the transfer of PCDD/Fs to terrestrial and aquatic ecosystems via deposition (Chao et al. 2004).

In order to measure the ambient air concentrations of PCDD/Fs and assess the air quality, active or passive air sampling techniques are used to collect the air samples. Passive air sampling techniques have been developed in recent years (Clarkson et al. 2002; Wennrich et al. 2002; Klanova et al. 2006). Passive air samplers are based on the difference in chemical potential of the analytes between the sampled medium and the collection medium. Because of their low cost and simple manipulation, polyurethane foam (PUF) disks are especially attractive (Shoeib and Harner 2002; Mari et al. 2008). However, compared to active sampling, passive air sampling is not capable of collecting sufficient air in only a few days to be used for detection of compounds that are present in the atmosphere at low concentrations. As conventional stationary active sampling techniques, high-volume active air samplers have been used for decades to monitor atmospheric concentrations of persistent organic pollutants (POPs). This system uses a pump to draw air through a glass fiber filter to collect particulate species, and then an absorbent trap to collect gaseous phase species. However, this type of sampling can be costly and not always feasible. These stationary samplers are usually fixed onsite where power supply is necessary, and cannot provide an overall air pollution level for a large area.

Air filter set is a necessary component of automobile. It is usually composed of polyurethane and other fiber-like materials. The air filter set of an automobile is usually placed inside a plastic or metal box connected to the throttle body with an intake tube. When the engine is working, air is taken into the cylinder and any dust in the air is retained on the air filter.

In this study, a novel mobile active sampling technique was developed to monitor POPs. Air filter set of 
automobiles was used to collect PCDD/Fs from the atmosphere over three different sampling periods. The sampling equipment was in constant motion throughout the zone, so the result was a reflection of the average pollution level in a large area. To the best knowledge, this is the first report of a mobile active air sampler for PCDD/Fs collection in ambient air. The automobile air filter set could be employed as a complementary tool for monitoring POPs in the atmosphere in metropolis.

\section{Materials and Methods}

Factory-installed automobile air intake filter sets (Beijing Hyundai Co., Product No. 28113-08000, China) on three vehicles (Hyundai Elantra 1.6 L, 2005 model) were employed for sampling PCDD/Fs in the air.

The air sample volume for each sampling period was estimated from fuel consumption. The air/fuel ratio is the mass ratio of air to fuel present during combustion. For gasoline fuel, the stoichiometric air/fuel ratio is approximately 14.7. For a car moving at approximately $60 \mathrm{~km} / \mathrm{h}$ with a fuel consumption of approximately $0.09 \mathrm{~L} / \mathrm{km}$, the intake airflow is estimated to be approximately $992 \mathrm{~L} / \mathrm{km}$ at standard temperature and pressure. The density of fuel ( $\rho_{\text {petro }}$ ) is assumed to be about $750 \mathrm{~g} / \mathrm{L}$, and the density of air $\left(\rho_{\text {air }}\right)$ is $1.293 \mathrm{~g} / \mathrm{L}$. The air sampling volume can be calculated by the following equation (Eq. 1:

$$
\begin{aligned}
\mathrm{V}_{\text {air }} & =\left(14.7 \times \rho_{\text {petro }} \times \mathrm{V}_{\text {petro }} \times \mathrm{s}\right) / \rho_{\text {air }} \\
& =\left(14.7 \times 750 \mathrm{~g} / \mathrm{L} \times \mathrm{V}_{\text {petro }} \times \mathrm{s}\right) / 1.293 \mathrm{~g} / \mathrm{L}
\end{aligned}
$$

$\mathrm{V}_{\text {air }}$ is the air sampling volume, $\mathrm{V}_{\text {petro }}$ is the petroleum consumption (L) per kilometer, and $\mathrm{s}$ is the driving distance $(\mathrm{km})$.

Samples were collected between April 28 and June 25, 2007. Detailed sampling information is given in Table 1. In order to evaluate the feasibility and effectiveness of the mobile active air sampler, three different sampling campaigns were conducted over the entire sampling period. The sampling volumes were approximately 500, 1,800 and $8,000 \mathrm{~m}^{3}$, respectively. All three test vehicles were driven through downtown Beijing during each sampling campaign. After a suitable interval, the used filter was replaced with a new clean filter, and the used filter was analyzed for PCDD/Fs. Dust collected on the used filter should reflect the average atmospheric PCDD/Fs concentrations over the entire period of use.

All filters were spiked with ${ }^{13} \mathrm{C}_{12}$-labeled $\mathrm{PCDD} / \mathrm{Fs}$ (Wellington Laboratories, Canada) before use, to monitor the performance of the sampling method. At the end of each sampling campaign, these filters were removed immediately, and wrapped with hexane rinsed aluminum foil.

All the organic solvents were pesticide residue grade from Fisher Co. (USA). Silica gel (100-200 mesh) was purchased from Qingdao Haiyang Chemical Co., China. Basic alumina was obtained from Shanghai Wusi Chemical Co., China. Calibration standard solutions, ${ }^{13} \mathrm{C}$-labeled surrogate standards and injection standards complying with EPA method 1613B for PCDD/Fs analysis were purchased from Wellington Laboratories (Canada).

Each used filter was cut into $2 \mathrm{~cm}$ size squares and extracted together with dust collected during disposal of filter by accelerated solvent extraction (ASE 300 from Dionex USA). The extracts were washed with sulfuric acid and purified on a multi-layer silica gel, and then passed through a basic alumina column. The PCDD/Fs fraction was concentrated almost to dryness under a gentle nitrogen stream. The final extract was spiked with the isotope labeled internal standard and then analyzed by high resolution gas chromatography (Agilent 6890) coupled with high resolution mass spectrometry (Waters Autospec Ultima) to determine the recovery efficiencies achieved for the ${ }^{13} \mathrm{C}_{12}$-labeled injection internal standards. Exactly $1 \mu \mathrm{L}$ of concentrated extract was injected with a CTC PAL auto-sampler in splitless mode into a DB-5MS column

\begin{tabular}{|c|c|c|c|c|c|}
\hline $\begin{array}{l}\text { Sample } \\
\text { ID }\end{array}$ & $\begin{array}{l}\text { Sampling } \\
\text { periods }\end{array}$ & $\begin{array}{l}\text { Vehicles } \\
\text { no. }\end{array}$ & $\begin{array}{l}\text { Driving } \\
\text { distance }(\mathrm{km})\end{array}$ & $\begin{array}{l}\text { Petro } \\
\text { consumption (L) }\end{array}$ & $\begin{array}{l}\text { Sampling } \\
\text { volume }\left(\mathrm{m}^{3}\right)\end{array}$ \\
\hline M1-1 & \multirow[t]{3}{*}{ April 28-29 } & $\mathrm{T} 1$ & 579 & 52 & 445 \\
\hline M1-2 & & $\mathrm{T} 2$ & 669 & 60 & 514 \\
\hline M1-3 & & $\mathrm{T} 3$ & 704 & 63 & 542 \\
\hline M2-1 & \multirow[t]{3}{*}{ April 30-May 6} & $\mathrm{~T} 1$ & 2,354 & 212 & 1,810 \\
\hline M2-2 & & $\mathrm{T} 2$ & 2,957 & 266 & 2,274 \\
\hline M2-3 & & $\mathrm{T} 3$ & 1,927 & 173 & 1,482 \\
\hline M3-1 & \multirow[t]{3}{*}{ May 25-June 25} & $\mathrm{~T} 1$ & 11,622 & 1,045 & 8,932 \\
\hline M3-2 & & $\mathrm{T} 2$ & 10,512 & 946 & 8,085 \\
\hline M3-3 & & T3 & 10,701 & 963 & 8,231 \\
\hline
\end{tabular}

Table 1 Sampling information 
Fig. 1 The amount of PCDD/ Fs in samples with different sampling volume. M1 represent the average values of M1-1, M1-2 and M1-3, M2 represent the average values of M2-1, M2-2 and M2-3, and M3 represent the average values of M3-1, M3-2 and M3-3, respectively

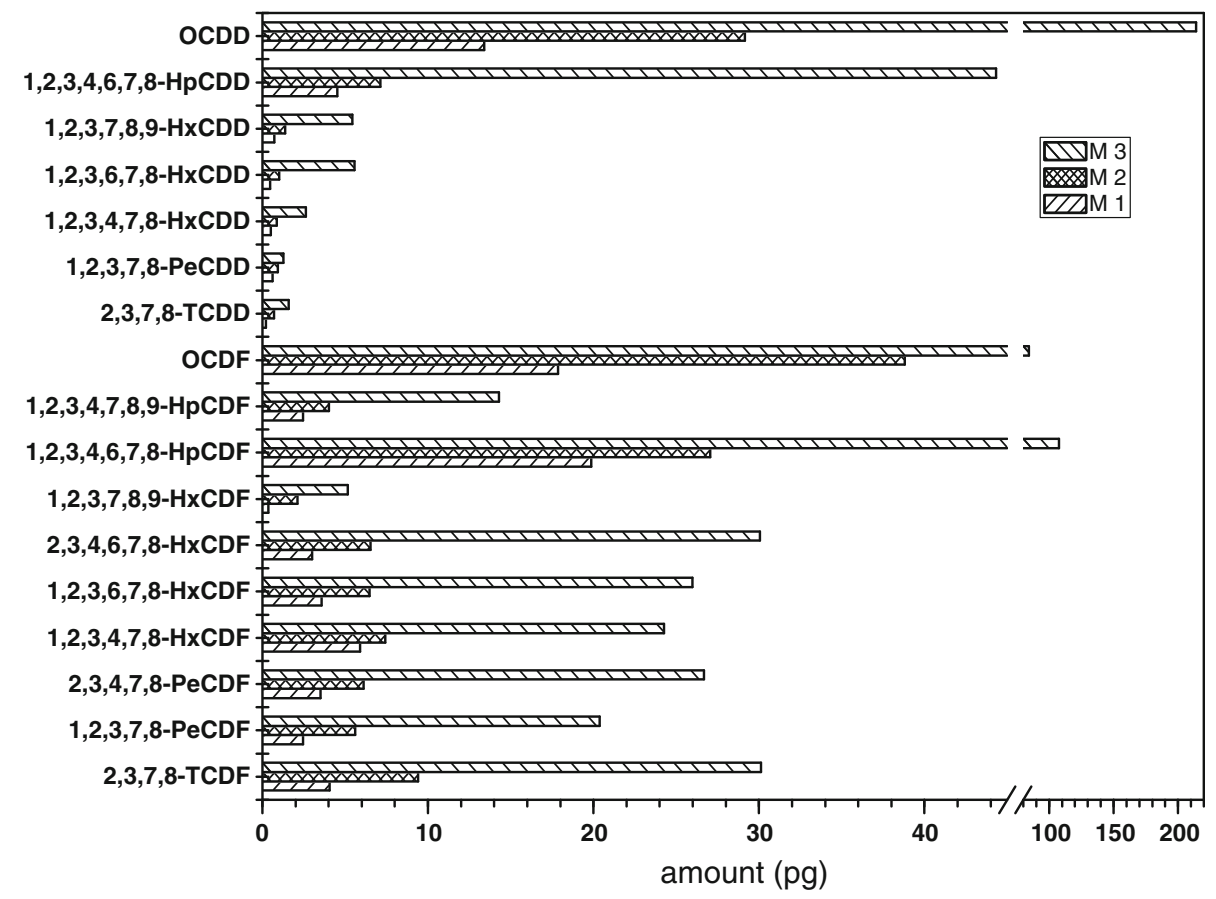

$(60 \mathrm{~m} \times 0.25 \mathrm{~mm}$ i.d. $\times 0.25 \mu \mathrm{m})$. The carrier gas was helium at a flow rate of $1.2 \mathrm{~mL} / \mathrm{min}$. The mass spectrometer was equipped with an electron ionization (EI) source and operated in positive ion mode ( $38 \mathrm{eV}$ electron energy) and selected ion monitoring (SIM) mode, at resolving power $\mathrm{R}>10,000$.

For the purpose of quality control, all samples were spiked with labeled compounds to monitor the performance of the method. Air filter blank and laboratory blank samples were incorporated in the analytical procedure. There was no apparent difference between the filter blanks and laboratory blanks. OCDD and 1,2,3,4,6,7,8-HpCDD were the most prevalent contaminants for $\mathrm{PCDD} / \mathrm{Fs}$ in the blanks. However, the concentrations of OCDD and $1,2,3,4,6,7,8$-HpCDD in the blanks corresponded to $<5 \%$ of the concentrations analyzed in the air samples. The limit of detection (LOD) for PCDD/Fs was defined as the signalto-noise ratio more than three times the average baseline variation.

\section{Results and Discussion}

According to the US EPA TO-9A method (US EPA 1999), the recommended sampling time for a stationary high volume active sampler is 1 days with a total volume in the range of $325-400 \mathrm{~m}^{3}$. Significant loss was not detected when duplicate samplers were operated for 7 days and sampled 2,660 $\mathrm{m}^{3}$ of ambient air. To verify the hypothesis that the automotive air filter set can retain and accumulate $\mathrm{PCDD} / \mathrm{Fs}$ from the atmosphere, the active sampler was used to take ambient air samples of 500, 1,800, and $8,000 \mathrm{~m}^{3}$. Figure1 shows the amount of PCDD/Fs detected in three samples with different volumes. The seventeen 2,3,7,8-substituted congeners were found in every sample. The mass of these congeners collected on the air filter increased with the volume of the air sample.

Experiments were conducted to determine the collection efficiencies and evaluate the incidence of PCDD/F congeners passing through the filter. The recoveries of the labeled PCDD/F congeners spiked into the air filter are shown in Fig. 2. When the sampling volume was about $500 \mathrm{~m}^{3}$, the average recoveries for the PCDD/F congeners were $>62.3 \%$ with relative standard deviation (RSD) $<17.3 \%$. When the volume increased to $1,800 \mathrm{~m}^{3}$, the average recoveries of PCDD/Fs increased to $>64.5 \%$ with RSD $<19.3 \%$. However, when the sampling volume increased to $8,000 \mathrm{~m}^{3}$, the average recoveries of the $\mathrm{PCDD} / \mathrm{Fs}$, especially those of $1,2,3,4,7,8-\mathrm{HxCDF}$ and 1,2,3,6,7,8-HxCDF and 1,2,3,4,6,7,8-HpCDF, decreased dramatically with RSD ranging from $8.5 \%$ to $64.5 \%$. This indicates that larger volume $\left(8,000 \mathrm{~m}^{3}\right)$ is not acceptable for quantification of PCDD/Fs in atmosphere. The volume of $1,800 \mathrm{~m}^{3}$ in this study was collected in 6 days and produced acceptable mean recoveries for all congeners.

In order to evaluate the feasibility of the novel mobile air sampler, the levels of PCDD/Fs in atmosphere collected by the automotive filter set were compared with those monitored by the stationary high volume active samplers in Beijing (Li et al. 2008) (Fig. 3). The data obtained by the mobile active air samplers agreed with that obtained by the stationary high volume samplers. This suggests that this 
Fig. 2 The average recoveries of PCDD/Fs and RSD compared with different sampling volumes. M1 represent the average values of M1-1, M1-2 and M1-3, M2 represent the average values of M2-1, M2-2 and M2-3, and M3 represent the average values of M3-1, M3-2 and $\mathrm{M} 3-3$, respectively
Fig. 3 Comparison of PCDD/ Fs profiles by mobile sampler and stationary high-volume sampler. M1 represent the average values of M1-1, M1-2 and M1-3
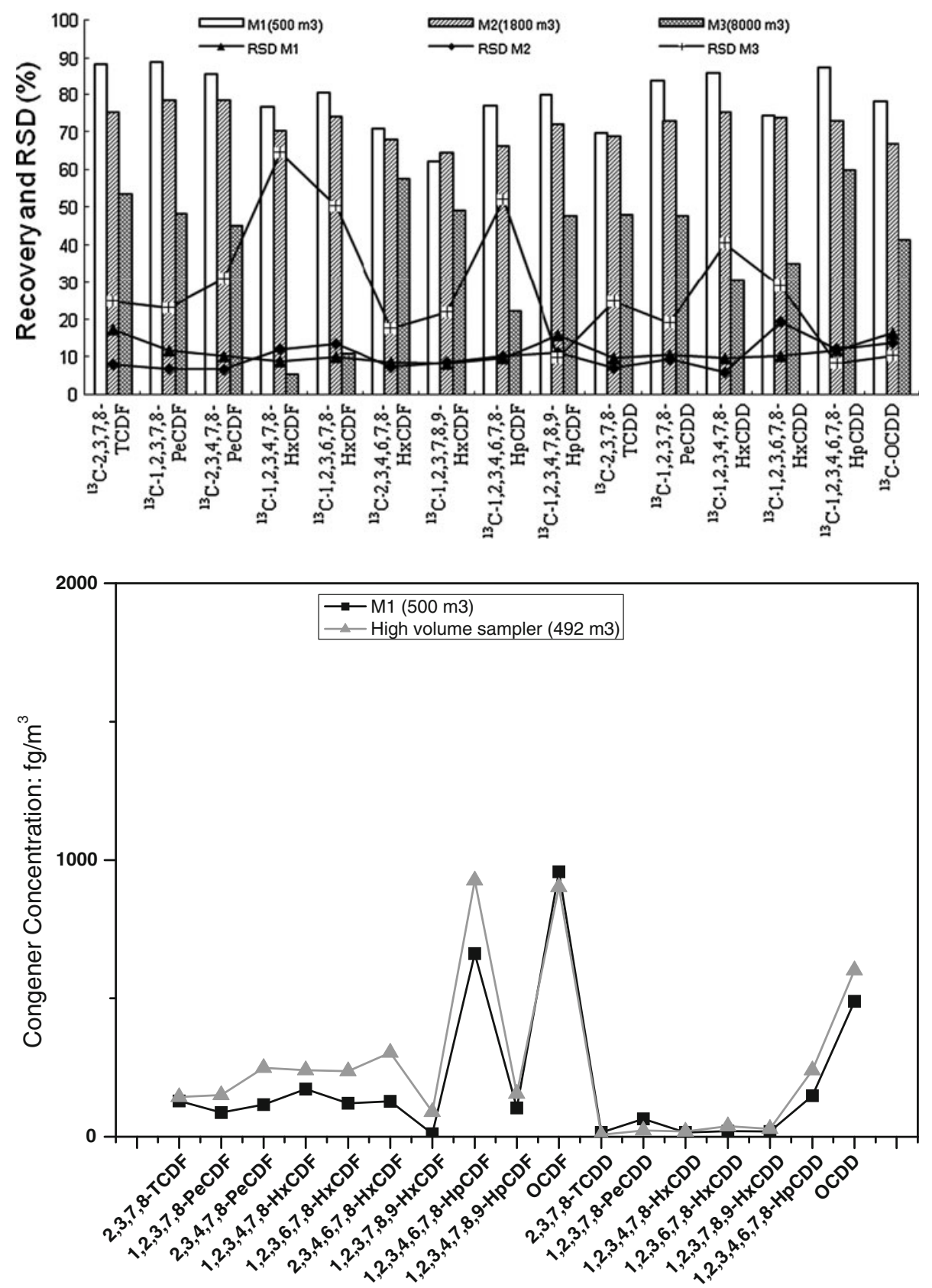

mobile active air sampler could be used as a complementary tool for monitoring POPs in a large area in metropolis.

In summary, an automobile air filter set was used to collect PCDD/Fs from the atmosphere. PCDD/Fs recoveries met US EPA Method TO-9 with a moderate volume. Furthermore, PCDD/F concentrations were comparable with those obtained by stationary high volume sampler. This mobile sampler can be used to collect samples that reflect the average pollution levels in large areas.

Acknowledgments The work was supported by National High-tech R\&D Program of China (2007AA061602, 2007AA06A407, 2009AA061603).

\section{References}

Chang H, Wang S, Wang Y, Li H, Wang L (2010) Contributions of dry and wet depositions of polychlorinated dibenzo-p-dioxins and dibenzofurans to a contaminated site resulting from a penetachlorophenol manufacturing process. Environ Monit Assess (in press). doi:10.1007/s10661-010-1545-X

Chao MR, Hu CW, Chen YL, Chang-Chien GP, Lee WJ, Chang LW, Lee WS, Wu KY (2004) Approaching gasparticle partitioning equilibrium of atmospheric PCDD/Fs with increasing distance from an incinerator: measurements and observations on modeling. Atmos Environ 38:15011510

Clarkson PJ, Larrazabal-Moya D, Staton I, McLeod CW, Ward DB, Sharifi VN, Swithenbank J (2002) The use of tree bark as a 
passive sampler for polychlorinated dibenzo-p-dioxins and furans. Int J Environ Anal Chem 82:843-850

Klanova J, Kohoutek J, Hamplova L, Urbanova P, Holoubek I (2006) Passive air sampler as a tool for long-term air pollution monitoring: Part 1. Performance assessment for seasonal and spatial variations. Environ Pollut 144:393-405

Li YM, Jiang GB, Wang YW, Cai ZW, Zhang QH (2008) Concentrations, profiles and gas-particle partitioning of polychlorinated dibenzo-p-dioxins and dibenzofurans in the ambient air of Beijing, China. Atmos Environ 42:2037-2047
Mari M, Schuhmacher M, Feliubadalo J, Domingo JL (2008) Air concentrations of PCDD/Fs, PCBs and PCNs using active and passive air samplers. Chemosphere 70:1637-1643

Shoeib M, Harner T (2002) Characterization and comparison of three passive air samplers for persistent organic pollutants. Environ Sci Technol 36:4142-4151

Wennrich L, Popp P, Hafner C (2002) Novel integrative passive samplers for the long-term monitoring of semivolatile organic air pollutants. J Environ Monit 4:371-376 